\title{
Picosecond laser ultrasonics for imaging of transparent polycrystalline materials compressed to megabar pressures
}

\author{
Maju Kuriakose a , Samuel Raetz ${ }^{\text {a }}$, Nikolay Chigarev ${ }^{a}$, Sergey M. Nikitin ${ }^{\mathrm{a}}$, Alain Bulou ${ }^{\mathrm{b}}$, Damien Gasteau ${ }^{\mathrm{a}, \mathrm{c}}$, \\ Vincent Tournat $^{\mathrm{a}}{ }^{\text {, Bernard Castagnede }}{ }^{\mathrm{a}}$, Andreas Zerr ${ }^{\mathrm{d}, *}$, Vitalyi E. Gusev ${ }^{\mathrm{a}, *}$ \\ a LAUM, UMR-CNRS 6613, Université du Maine, Le Mans, France \\ b IMMM, UMR-CNRS 6283, Université du Maine, Le Mans, France \\ ${ }^{\mathrm{C}}$ CEA Saclay DIGITEO Labs, Gif-Sur-Yvette, France \\ ${ }^{\mathrm{d}}$ LSPM, UPR-CNRS 3407, Université Paris Nord, Villetaneuse, France
}

\section{A R T I C L E I N F O}

\section{Article history:}

Received 30 September 2015

Received in revised form 1 March 2016

Accepted 8 March 2016

Available online 18 March 2016

\section{Keywords:}

Laser ultrasonics

Picosecond ultrasonic interferometry

Time-domain Brillouin scattering

3D imaging

High pressures

\begin{abstract}
A B S T R A C T
Picosecond laser ultrasonics is an all-optical experimental technique based on ultrafast high repetition rate lasers applied for the generation and detection of nanometric in length coherent acoustic pulses. In optically transparent materials these pulses can be detected not only on their arrival at the sample surfaces but also all along their propagation path inside the sample providing opportunity for imaging of the sample material spatial inhomogeneities traversed by the acoustic pulse. Application of this imaging technique to polycrystalline elastically anisotropic transparent materials subject to high pressures in a diamond anvil cell reveals their significant texturing/structuring at the spatial scales exceeding dimensions of the individual crystallites.
\end{abstract}

(c) 2016 Elsevier B.V. All rights reserved.

\section{Introduction}

Picosecond laser ultrasonics, developed since the pioneering works of Maris and co-workers [1], is a technique that uses ultra-short pump laser pulses to generate coherent acoustic pulses and detect them by time-delayed ultra-short probe laser pulses while they propagate through a transparent medium or while they reach surfaces/interfaces. For launching acoustic pulses into optically transparent samples the latter can be covered with thin layers of light absorbing materials and even single layer light absorbers [2]. The length of the laser-generated pulses depend in general on the duration of the pump laser pulses, pump light penetration depth in the material and/or the thickness of the light-absorbing opto-acoustic transducer. Through the minimization of these factors it is possible to generate coherent acoustic pulses with a spatial length of nanometers order, which makes them suitable for imaging purposes at nanoscale. The contrast in imaging of materials by picosecond laser ultrasonics could be of different physical origins. It could be caused either by the spatial inhomogeneity of the photoacoustic generation process (optoacoustic conversion),

\footnotetext{
* Corresponding authors.

E-mail addresses: zerr@univ-paris13.fr (A. Zerr), vitali.goussev@univ-lemans.fr (V.E. Gusev).
}

or by the influence of the material inhomogeneity on the propagation of the coherent acoustic pulses, or by the spatial inhomogeneity of the acousto-optic detection process, or by a combination of those: thermal, thermo-elastic and optical parameters of the sample material (the latter at the pump laser wavelength) are influencing thermoelastic optoacoustic conversion; acoustic parameters of the material (such as sound velocity and acoustic impedance) are influencing propagation of the acoustic pulses; and, finally, acoustical, optical and acousto-optical parameters of the sample material at the wavelength of the probe laser light are influencing the detection process. Accordingly, imaging with picosecond laser ultrasonics is expected to be sensitive to multiple parameters of the examined medium. Because of this and of the potential of nanometric in-depth spatial resolution, picosecond ultrasonic technique has been widely used for numerous imaging applications. The principles of these imaging techniques, which are different depending on the nature of inhomogeneity that provides the image contrast, are briefly reviewed in the following.

In sonar and medical ultrasound techniques, a piezo transducer is used to generate ultrasound to image acoustic inhomogeneities. The contrast in such imaging is based on the ultrasound reflections caused by acoustical inhomogeneities (spatial variations in acoustic impedance). The similar imaging can be achieved with picosecond laser ultrasonics, where laser replaces the piezoelectric 
transducers for the generation and detection of hypersound. For instance, the comparison in a wide frequency band of the reflection coefficient of nanoacoustic waves at the $\mathrm{GaN} / \mathrm{H}_{2} \mathrm{O}$ interface with that at the GaN/air interface provided indication on the structuring of the $\mathrm{GaN} / \mathrm{H}_{2} \mathrm{O}$ interface at nanoscale [3]. Later, through a comparison of the hypersound reflection coefficient at an $\mathrm{Al}_{2} \mathrm{O}_{3}$ /water and at $\mathrm{Al}_{2} \mathrm{O}_{3}$ /air interfaces the profiles of density, modulus and viscosity of water were reconstructed at depths up to $1 \mathrm{~nm}$ from the interface. It was demonstrated that liquid-water density in the vicinity (few angstroms) of the $\mathrm{Al}_{2} \mathrm{O}_{3}$ /water interface is up to five times larger than in bulk liquid-water [4]. Based on the same acoustic reflection coefficient wide-frequency-band analysis, acoustic images of single biological cells were recently reported, allowing analysis of the cell/substrate adhesion at nanoscales [5]. In these applications, the small length of laser-generated acoustic pulses is advantageously used to improve the in-depth spatial resolution of the imaging.

On the other hand, the so-called photoacoustic imaging [6,7] is mostly based on the contrast due to possible spatial inhomogeneity of pump laser radiation absorption in optically transparent/diffusive multiphase media. Though this was initially developed for medical purposes [6-8] using, in particular, contrast in light absorption between blood containing vessels and tissue, the technique is also receiving a growing attention for industrial applications in solid media [9-13] where the contrast in optoacoustic conversion is due to spatial inhomogeneity in chemical composition of the samples. Regardless of its potential to produce images combining high optical contrast and high acoustic resolution, the technique has not been advanced until now to the nanoscales, although at depths shallower than $1 \mathrm{~mm}$, optical-resolution photoacoustic microscopic system was developed [8].

Finally, while dealing with optically transparent media, it is possible to realize imaging based on the spatial contrast in acousto-optic detection process. This imaging is sensitive to some additional parameters of the media, which cannot be probed by the two above described approaches. This type of imaging can be achieved with ultrafast lasers by the technique that is known under the name of the picosecond acoustic interferometry $[14,15]$ or time-resolved/time-domain Brillouin scattering. Indeed, when a laser-generated picosecond coherent acoustic pulse propagates in a medium, it induces through the acousto-optic effect a local change in the density and, accordingly, in the refractive index of the medium proportional to the acoustic strain. This causes a local reflection of the probe light for which the medium is transparent [see the paths of the pump (in blue) and probe (in red) optical rays in the sample in the left part of Fig. 1]. The name "interferometry" comes from the fact that, at the photodetector, the reflections of the probe laser beam from different stationary surfaces and interfaces of the tested sample interfere with the probe laser reflections from the moving coherent acoustic pulse. The scattering of probe in the considered case is the Brillouin scattering of light by coherent acoustic waves (coherent phonons). Similar to the Brillouin scattering by thermal incoherent phonons, the process is controlled, in addition to acoustic parameters of the material (sound velocity and acoustic impedance, or equivalently material density and elastic moduli), by the optical refractive index and the acousto-optic (photo-elastic) tensor that defines efficiency of light interaction with the sound pulse. The transient signal detected in picosecond ultrasonic interferometry thus contains, at each moment of time, information on the local elastic, optical and elasto-optical properties of the tested material at the position where the ultrashort acoustic pulse is located during its propagation in the sample. The detected interference signal has commonly the form of a quasi-sinusoidal oscillation (the so-called Brillouin oscillation, see Fig. 2), where the information on the local parameters of the inhomogeneous media traversed by the coherent acoustic pulse is contained both in the amplitude and in the phase (frequency) of the oscillation [16]. This technique of picosecond ultrasonic interferometry was successfully applied for the depth profiling of material properties with nanometric spatial resolution at ambient conditions [17-19] and has been recently extended to probe material properties at megabar pressures [20]. The imaging was based on the analysis of the temporal evolution of either the Brillouin oscillation frequency [17] or the Brillouin oscillation amplitude [18], or of the both [19]. The in-depth spatial resolution of about $40 \mathrm{~nm}$ was achieved in low- $k$ nanoporous sub- $\mu \mathrm{m}$ thick films [17,19] and of about $30 \mathrm{~nm}$ in ion-irradiated semiconductor [18]. Three-dimensional imaging by this technique of a single biological cell, which allows average measurements of sound velocities and attenuation in the cells, was also reported [21].

In this paper, the information on the picosecond acoustic interferometry, on the required signal processing, and on their applicability to image transparent materials compressed up to megabar pressures in a diamond anvil cell (DAC) are presented. The purposes of conducting high spatial resolution imaging at megabar pressures are to measure accurately local mechanical properties of materials under extreme conditions as well as to get spatially resolved images of texturing (ordering of nano- and

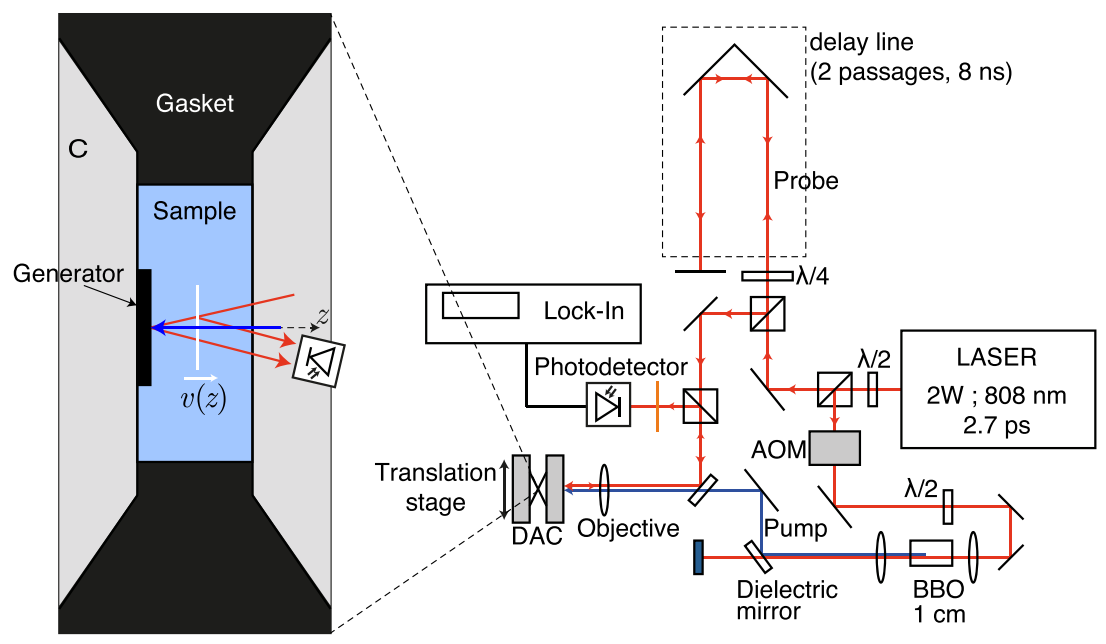

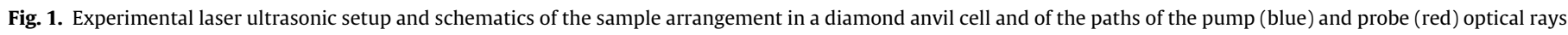
in the transparent sample. (For interpretation of the references to color in this figure legend, the reader is referred to the web version of this article.) 

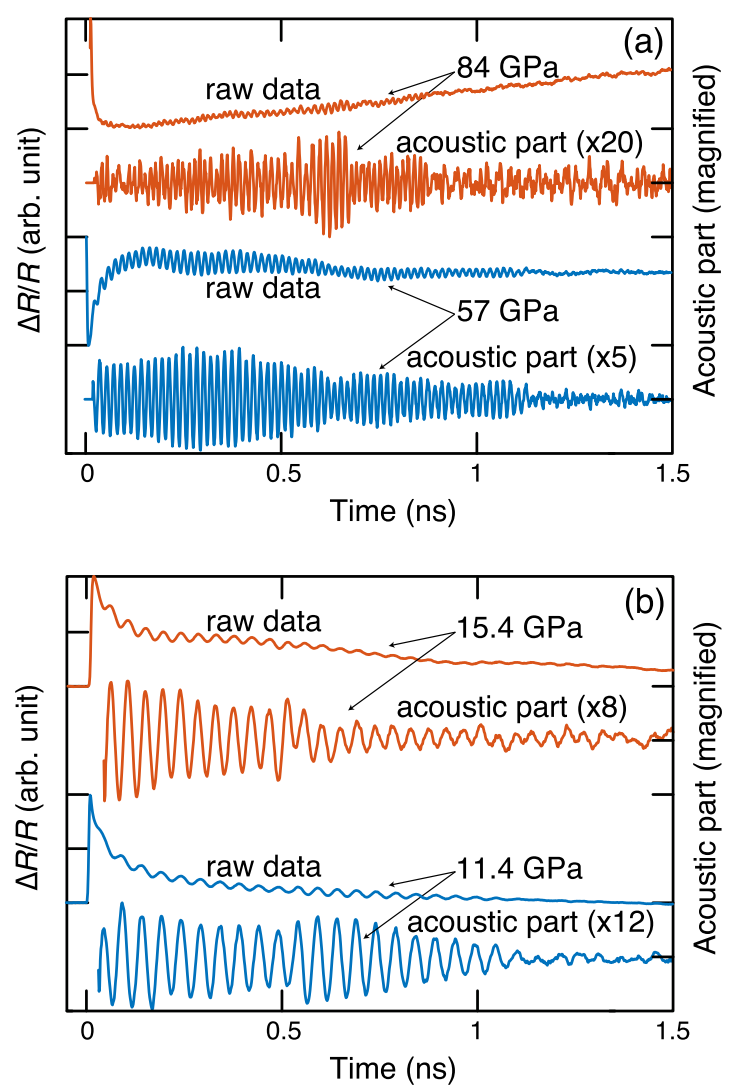

Fig. 2. Typical transient reflectivity signals (each normalized to their maximum) and their acoustic parts (magnified for better visibility) detected for: (a) the $\mathrm{H}_{2} \mathrm{O}$ ice sample compressed to $57 \mathrm{GPa}$ (in blue, bottom) and to $84 \mathrm{GPa}$ (in orange, top); (b) the polycrystalline argon sample compressed to $11.4 \mathrm{GPa}$ (in blue, bottom) and to 15.4 GPa (in orange, top) pressures. (For interpretation of the references to color in this figure legend, the reader is referred to the web version of this article.)

micro-crystallites at the scales exceeding dimensions of individual crystallites) in compressed polycrystalline materials. Both of these purposes cannot be achieved with the classical frequency-domain Brillouin scattering technique because the latter gives access only to the parameter values averaged over the thickness of the sample that commonly exceeds several micrometers in the high-pressure experiments conducted in a DAC $[22,23]$.

Thus the sub-micrometer and even the nanometer in-depth spatial resolution that could be provided by the time-domain Brillouin scattering leads to an enhanced accuracy in determining elastic properties of compressed materials, which are of extreme interest for a few branches of natural sciences such as condensed matter physics, physics of the Earth and planetology, as well as for monitoring and predicting earthquakes and tsunamis or nuclear weapons test control. This motivated our efforts in the developments and the improvements of this imaging technique. In the following sections, firstly, the experimental setup together with the depth-profiling technique is described. Then the signal processing is presented and the lateral and the in-depth resolutions are discussed. Finally, some examples of imaging of $\mathrm{H}_{2} \mathrm{O}$ ice and solid argon compressed up to megabar pressures, at room temperature, are presented, compared and discussed.

\section{Experimental setup and imaging technique}

To reproduce extreme pressure conditions in laboratories, the most widely used tool is the diamond anvil cell (DAC). The device consists of two opposing diamond anvils with the culets compressing a sample in the center of a metal gasket (see left part in Fig. 1). The sample volume has the lateral size of typically $100-150 \mu \mathrm{m}$ and the thickness of a few tens of micrometers. Decreasing the distance between the anvils results in an increase of pressure in the confined volume. The pressure can be monitored by a change in fluorescence wavelength of Ruby grains embedded in the sample. Note that we used two different DACs for our two samples (water ice and argon). For water ice, the DAC model was Boehler-Almax Plate DAC [24] with diamond anvils having culets of $300 \mu \mathrm{m}$ in diameter. For argon, a homemade DAC of the piston-cylinder type with diamond anvils having culets of $350 \mu \mathrm{m}$ in diameter was used. The sample volume in the DAC was filled with condensed argon at room temperature using a gas-loading apparatus developed by one of the authors of the present paper (AZ) and built by the company TOP INDUSTRIE.

Since diamond anvils are transparent to visible light, this experimental device can be coupled with the picosecond acoustic technique in order to follow pressure-induced changes in the elastic properties of the light absorbing materials [25] and also of transparent materials if the light absorbing opto-acoustic transducer is placed in contact with the sample [20].

\subsection{Picosecond acoustic interferometry}

The picosecond laser ultrasonic interferometry or time domain Brillouin scattering (TDBS) is based on typical pump-probe configuration for transient reflectivity optical measurements, which is described in Fig. 1. A Ti:Saphire laser source (Spectra-Physics, $2 \mathrm{~W}, 808 \mathrm{~nm}$ ) delivering laser pulses of $2.7 \mathrm{ps}$ FWHM duration at a repetition rate of $80 \mathrm{MHz}$ was used. The laser beam was split into two parts with a polarizing cube. The first part was frequencydoubled in a $1 \mathrm{~cm}$-long BBO non-linear crystal and was used as the pump. The second part of the laser beam was used as the probe, delayed in time with respect to the pump beam by means of a mechanical delay line (Fig. 1). The pump was modulated at a frequency of $161.1 \mathrm{kHz}$ by an acousto-optic modulator (AOM, Fig. 1) for performing a synchronous detection of the probe light scattered by the sample with a lock-in amplifier. Using a $50 \times$ objective lens [for water sample: Olympus, numerical aperture (NA) 0.5 , working distance (WD) $10.5 \mathrm{~mm}$; for argon sample: Mitutoyo, NA 0.42, WD $20.5 \mathrm{~mm}$ ], pump and probe were then focused to a circular spot on the surface of an optoacoustic generator (e.g., absorbing material such as Fe or $\mathrm{Zn}$ foils) compressed together with the transparent material of interest in the DAC. The pump pulses absorbed in the metallic opto-acoustic transducer generate, through the thermo-elastic effect, acoustic pulses that propagate both inside the generator and in the transparent medium. These coherent acoustic pulses composed of coherent acoustic phonons of $\mathrm{GHz}$ frequencies can then be detected by the probe laser pulses.

Detection of the acoustic pulses happens in such a way that, at the photodetector, the reflected probe laser pulses from various stationary material boundaries interfere with their weak reflections from the propagating acoustic pulses (see Fig. 1). The interference is either constructive or destructive, thereby producing quasiperiodic (quasi-sinusoidal) variations in the reflectivity signal amplitude, i.e., the so-called time-domain Brillouin oscillation (Fig. 2), when the acoustic pulses are propagating with slowly varying velocity leading to quasi-linearly varying in time phase shift between the various reflections of the probe. Note that the reflections from stationary optical inhomogeneities were dominated in the current experiments by those from the surface of the optoacoustic generator (see the zoomed schema of the sample in a DAC in Fig. 1). 
The velocity $v$ of the acoustic wave can then be computed from the frequency of the Brillouin oscillations by [14,15,26]:

$$
f_{B} \cong \frac{2 n v \cos \theta}{\lambda_{0}},
$$

where $f_{B}, \lambda_{0}, n$ and $\theta$ are the Brillouin oscillation frequency, wavelength of the probe beam, refractive index of the sample material at this wavelength, and angle between the propagation directions of probe and the acoustic pulse, respectively. For the experiments with negligible changes in $n$ and if $\theta$ and probe wavelength are known from the optical schema, the sound velocity can be calculated directly from the measured $f_{B}$. Several measurements conducted at different angles of probe incidence provide opportunity to determine the local sound velocity and the optical refractive index simultaneously in cases where they are both importantly spatially inhomogeneous [19].

Both water and argon, the materials of our particular interest here, appear as polycrystalline solids when compressed above a few GPa in a DAC. Accordingly, when the photo-generated coherent acoustic pulse propagates along the DAC axis, orientation changes of the crystallites in these polycrystalline aggregates lead to changes in acoustic velocity. However, because both water ice and Argon are of cubic crystallographic symmetry under the pressures of our current experiments, optical refractive index $n$ in Eq. (1) is isotropic/scalar and does not depend on the orientation of the local crystallites in polycrystalline microscopically inhomogeneous samples. In addition, it has been demonstrated [20] that refraction of a coherent acoustic pulse at the boundaries between differently oriented crystallites/grains, caused by the anisotropy of the elastic moduli even in cubic crystallites, leads to variations of the angle between the acoustic and probe light beams that is negligible in comparison with the elastic anisotropy-related variations in sound velocity. Thus in our picosecond acoustic interferometry experiments, if the acoustic pulse propagates through crystallites with different orientations, only the value of sound velocity at Brillouin frequency will be encoded through Eq. (1) in $f_{B}$. So a measurement of the time dependency of the Brillouin oscillation frequency would allow probing the in-depth changes in sound velocity, thus providing information both on polycrystallinity of the sample and elastic inhomogeneities (texture) inside the sample at a spatial scale exceeding the dimensions of the individual grains (for example on orientation texturing of groups of crystallites). We describe one of the signal processing methods that could be applied to perform such a depth profiling in the following.

\subsection{Imaging technique}

\subsubsection{Depth profiling}

The frequency components of a temporal signal from a typical TDBS experiment can be brought out by applying the fast Fourier transform (FFT) to the signal. For this, initially, the experimental signals were filtered to remove the non-oscillating background caused by a transient heating of the sample and a transient optical reflectivity (see the acoustic parts of the signals in Fig. 2). Then the cleaned Brillouin oscillations were converted to their analytical form by applying Hilbert transformation. The cosines of the phase of the analytical signals are free of the variations in amplitudes, while the information on the varying frequencies is conserved. The FFTs were then applied on these "damping"-free signals to bring out the frequencies in it.

Yet, treating the entire signal at once gives the information of the various frequencies present in the complete signal, but do not give any information about the moment in time when they show up. Thus this type of signal processing will give information on the sample properties along the complete path of the acoustic pulse propagation in the sample without its in-depth resolution. In order to overcome this limitation, standard time-frequency analysis can be used with a short-time Fourier Transform (STFT), the window size at FWHM of which can be chosen as small as a single Brillouin cycle (if the signal quality permits), so that the frequency corresponding to the temporal window containing the treated Brillouin cycle can be obtained. Once the frequency is known and thereby the velocity [using Eq. (1)], the time axis can be converted into depth and thus the depth dependent velocity can be found. The resolution (temporal or in-depth) for the above-described method of the signal processing is thus governed by the choice of the temporal window duration. For example, by choosing the window duration equal to the period of the lowest acoustic frequency importantly contributing to the signal FFT performed over the complete signal, it is possible to realize the same spatial resolution in imaging along the complete path of acoustic pulse propagation. The spatial resolution in this case will be limited by the wavelength of phonon at the considered Brillouin frequency. Note that other methods of signal processing could lead to a better spatial resolution [17,19] that is potentially limited not by the acoustic wavelength at Brillouin frequency, which is in general of the order of the probe optical wavelength in the medium, but by the spatial scale of the important strain variations in the coherent acoustic pulse which plays the role of the width of the moving optical mirror.

The above-mentioned method of signal processing is well suited for signals containing one frequency component varying with time. Some issues can arise for signals containing two (or more) different frequency components simultaneously. If these components are close compared to the frequency resolution of the STFT, which is inversely proportional to the window size, the retrieved frequency will be a weighted average of the different individual frequencies. The only mean to detect close components would be to increase the window size, thus to decrease the temporal/indepth resolution. Yet, for signals containing different but well separated frequency components, an analysis of the individual peaks existing in the spectrogram can allow the independently retrieving of the different frequency components.

\subsubsection{Imaging and resolution}

Combining the above-mentioned depth profiling technique with lateral scans (translation stage of the DAC in Fig. 1), a 2dimensional (2D) imaging of the sample under study can be obtained. This can also be extended to a complete 3-dimensional (3D) image of the specimen by performing the 2D imaging for the sample position changing in the second lateral direction.

The imaging resolution depends mainly on two factors: (i) the duration of the temporal moving window for the FFT that controls the in-depth resolution as mentioned earlier, and (ii) the laser beam spot size, which controls the lateral resolution. The laser beam spot size at the focal plane of the objective, i.e. twice the beam waist $w_{0}$, is linearly proportional to the focal length of the objective. However, a limitation in decreasing the focal length arises because decreasing $w_{0}$ also decreases the Rayleigh range - the range where the laser beam radius remains smaller than $\sqrt{2} w_{0}$. If the Rayleigh range is too short, the probed volume in the sample will not be cylindrical in shape, which may lead to a change in the probed area as a function of the depth. Therefore the selection of the objective in our experiments depended on two key points: (i) the minimization of the beam waist, in order to have a good lateral resolution, and (ii) keeping the Rayleigh range larger than the thickness of the sample, so that the probed cross section remains approximately the same all along the thickness of the tested material.

In our experiments on water ice, where a $50 \times$ objective was used, the lateral surface area of the cylindrical volume (tested by 
overlapping the probe laser beam and the coherent acoustic beam) is determined to be approximately $10 \mu \mathrm{m}^{2}$ [20]. In the reported below experiments in $\mathrm{Ar}$ the correlation function of the pump and probe beams was measured to be about $2.7 \mu \mathrm{m}$ in diameter at FWHM of the highest change in optical reflection, measured when the pump and probe pulses coincide in time. This provides an estimate of $5.7 \mu \mathrm{m}^{2}$ for the cross section of the material volume tested in each lateral position.

\section{Some examples of imaging of highly-pressurized transparent materials}

Before presenting the experimentally obtained 2D images of water ice and solid argon, a short highlight on the main goals pursued by conduction of these experiments is proposed.

\subsection{Presentation of water and argon at megabar pressures}

Water is an important compound that supports life and stays also as a simplest example of hydrogen bonding, which explains the numerous investigations that are conducted on this compound all around the world. Water is known to transform through a variety of phases at different thermodynamic conditions (pressure and temperature). The pressures we have chosen, $57 \mathrm{GPa}$ and $84 \mathrm{GPa}$, represent the water ice sample, most probably, in its phase $\mathrm{X}$. We cannot exclude that at $57 \mathrm{GPa}$ the water ice is still in phase VII because position of the phase boundary between phases VII and $\mathrm{X}$, both having cubic crystal structure, is not well localized even at room temperature [27]. To give a milestone, the highest studied pressure ( $84 \mathrm{GPa}$ ) corresponds to the depth of about $2000 \mathrm{~km}$ in the Earth's interior [28].

For conducting high-pressure experiments, pressuretransmitting medium (PTM) are mandatory to obtain hydrostatic compression of the compound under study. Among others, argon is widely used as PTM because of its soft nature and chemical inertness. However it was reported that argon starts to become nonhydrostatic at pressures above $30 \mathrm{GPa}$ [29]. More interesting are reports on a significant elastic anisotropy of solid argon starting from the pressure of its solidification of $1.35 \mathrm{GPa}$ even though its crystal structure is cubic $[29,30]$. Such elastic anisotropy, combined with non-hydrostaticity, could strongly affect the sound velocity values derived from the classical Brillouin spectroscopic measurements performed for a sample compressed in a DAC. The main reason is the absence in such experiments of any information on preferred orientations of argon crystallites, texture and/or distribution of elastic inhomogeneities in the sample volume. Pressure distribution measurements can also not provide information on the orientation of the crystallites and the texturing/ordering of the groups of the crystallites. The proposed imaging based on picosecond ultrasonic interferometry can overcome these limitations and shed light on in-depth and in-plane distribution of inhomogeneities in solid argon compressed in a DAC with sub-micrometric to, potentially, nanometric resolution. The chosen pressures to analyze the hydrostaticity of solid argon are 11.4 and $15.4 \mathrm{GPa}$.

It will be now described how we have used the experimental setup and the explained signal processing to image inhomogeneities in two compressed transparent materials: water ice and solid argon, both of which are in polycrystalline form at pressures above $10 \mathrm{GPa}$. In the following, the obtained images for the water ice and solid argon are presented and discussed.

\subsection{Imaging experiments}

\subsubsection{Water compressed to $57 \mathrm{GPa}$ and $84 \mathrm{GPa}$}

Change of the sound frequencies as a function of depth at several lateral positions are displayed in Fig. 3 for the water ice sample at two pressures: $57 \mathrm{GPa}$ [(a) and (b)] and $84 \mathrm{GPa}[(\mathrm{c})$ and (d)]. The lateral scans are accumulated with a step of $2 \mu \mathrm{m}$ $(1 \mu \mathrm{m})$ for $57 \mathrm{GPa}(84 \mathrm{GPa})$ along the surface of the optoacoustic transducer. Thicknesses of the ice samples at $57 \mathrm{GPa}$ and $84 \mathrm{GPa}$ were measured to be $14.4 \mu \mathrm{m}$ and $13.5 \mu \mathrm{m}$, respectively, using the interference patterns generated by illumination with the broadband light. The thicknesses were also determined from the time of propagation of the acoustic pulse between the generator and the opposite diamond anvil culet. A higher precision in the depth calculations was obtained where the time when the acoustic pulse reaches the diamond culet was chosen as the starting depth value. Then the depth coordinate has been reversely calculated back to the generator position. This helps to eliminate the uncertainties in the depth caused by generator thickness irregularities and lower precision of our signal processing technique at short distances from the opto-acoustic transducer. At such distances there is a significant contribution to transient reflectivity related to a direct dependence of the reflectivity on the temperature in the generator and the sample [20]. We used a color scale to depict the 2D sound frequency distribution in the ice samples at $57 \mathrm{GPa}$ and $84 \mathrm{GPa}$, collected with two different in-depth resolutions (Fig. 3).

The frequency values in the images were obtained by applying the previously presented STFT processing. The chosen FWHM of the Hann window was 70 ps (3-4 oscillations of the longest Brillouin cycle) in Fig. 3(a) and (c), and $17.5 \mathrm{ps}$ (nearly equal to the length of the longest Brillouin cycle) in Fig. 3(b) and (d). For the $84 \mathrm{GPa}$ sample, these time windows correspond to in-depth resolutions of about $0.9-1.2 \mu \mathrm{m}$ for $70 \mathrm{ps}$ window [Fig. 3(c)] and $0.22-0.28 \mu \mathrm{m}$ for $17.5 \mathrm{ps}$ window [Fig. 3(d)]. The characteristic dimension of individual crystallites in both polycrystalline ice samples was estimated to be around $0.45 \mu \mathrm{m}$ using XRD techniques [20]. Considering the best indepth resolution controlled by the chosen signal processing and the lateral dimensions of the pump-probe overlap at FWHM of $10 \mu \mathrm{m}^{2}$, the minimal opto-acoustically tested volume was about $2.6 \mu^{3}$. This means that the frequencies are averaged at least over approximately 30 individual crystallites. The images at $84 \mathrm{GPa}$ show clearly a low frequency layer in the depth region of $7-11 \mu \mathrm{m}$ [Fig. 3(c) and (d)], while for the 57 GPa sample the in-depth texturing/structuring of the water ice is less pronounced. The maximum changes of the Brillouin frequency relative to the average level revealed in the experiments with water ice are approximately $\pm 26 \%$ [20]. The local axial changes in frequency cannot be related to the axial pressure gradients inside the sample because this would also require large pressure gradients in the lateral direction, much higher than the observed maximal difference of about $10 \mathrm{GPa}$. The in-depth variation in frequencies thus reveals that the phase $\mathrm{X}$ of water ice is strongly anisotropic and the variation of frequencies is dominated by the variation in the orientation of individual crystallites or groups of crystallites [20]. The velocity variations between the adjacent maxima to minima were found to approach about $\pm 13 \%$, which is rather close to the theoretically predicted value, of $\pm 16 \%$, reported in Ref. [31]. The observed lowest frequency in our sample can be attributed to the orientation of crystallites (texturing) in the direction $\langle 100\rangle$ at which the sound velocity is the lowest compared to other orientations: $\langle 110\rangle$ and $\langle 111\rangle$. Similarly, the highest frequency can be attributed to the fastest $\langle 111\rangle$ direction. Taking this fact into account we can conclude that the sample is stronger textured at $84 \mathrm{GPa}$ than at $57 \mathrm{GPa}$. From the analysis of Fig. 3(b) and (d) where the in-depth resolution is the highest, it can be deduced that the samples contain elastically homogeneous mesoscopic crystallite groups up to $1 \mu \mathrm{m}$ lateral size and about $0.3-0.6 \mu \mathrm{m}$ thickness [20]. 

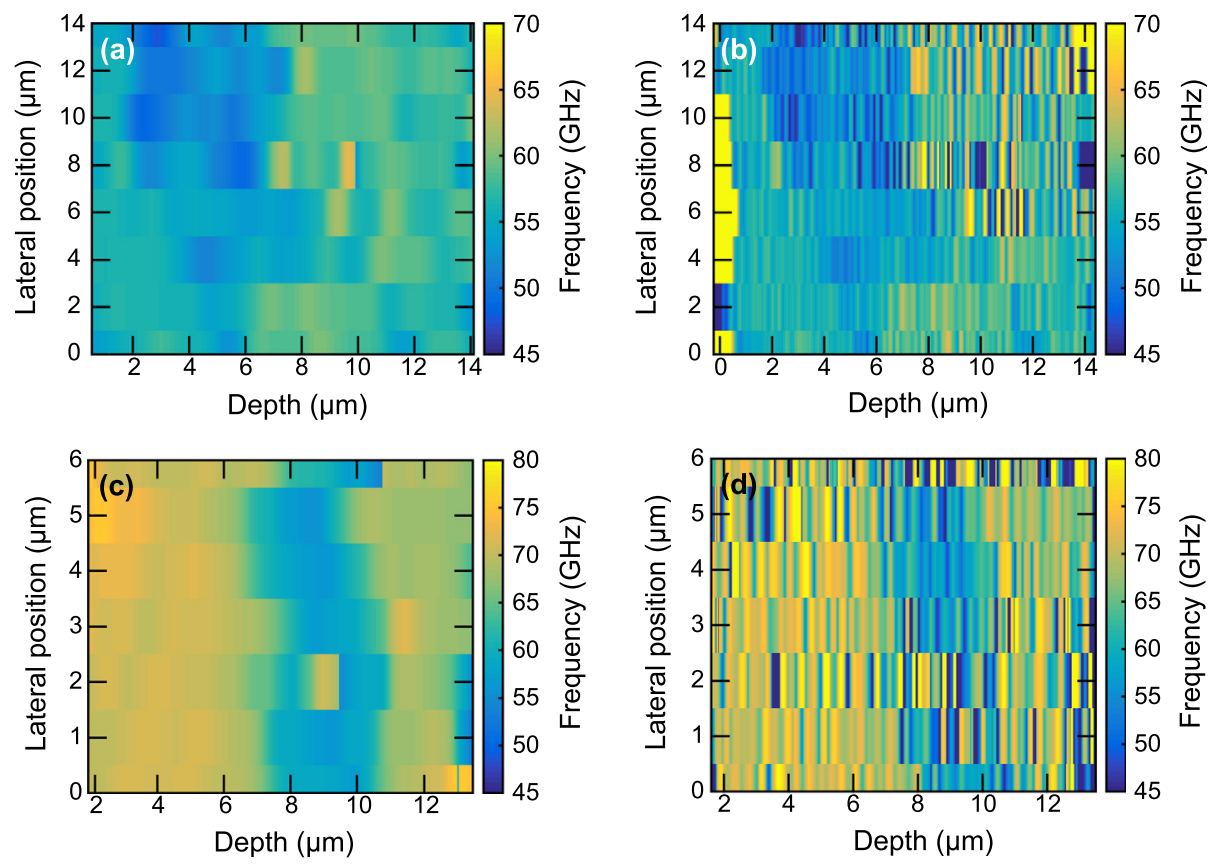

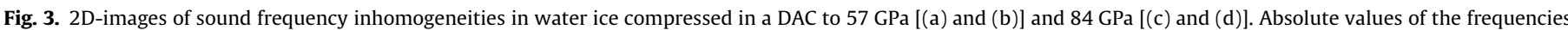

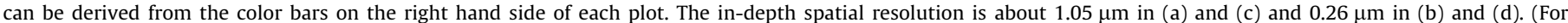
interpretation of the references to color in this figure legend, the reader is referred to the web version of this article.)
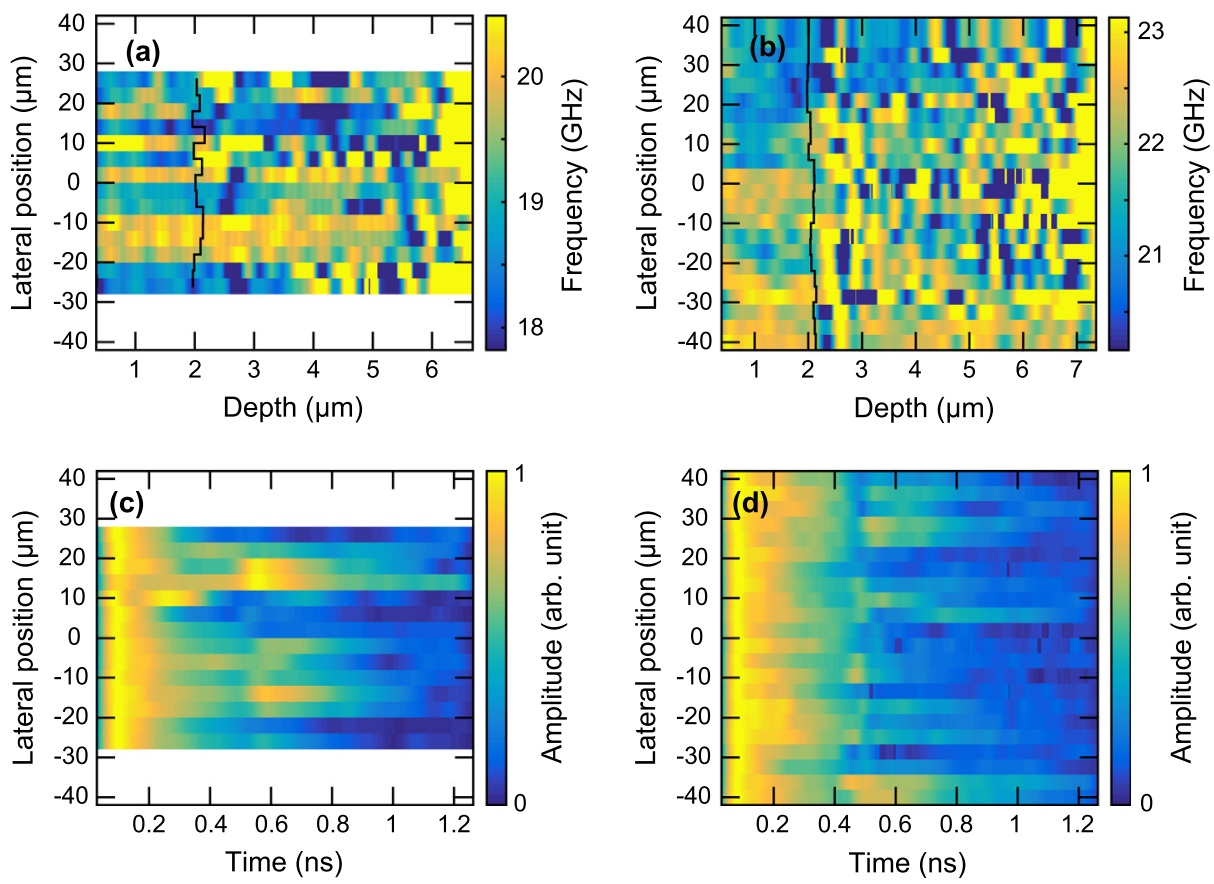

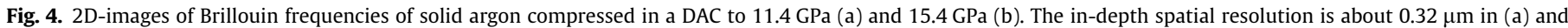

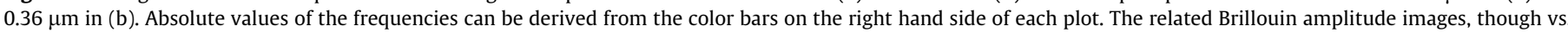

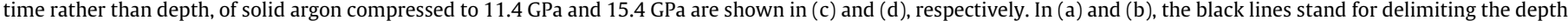

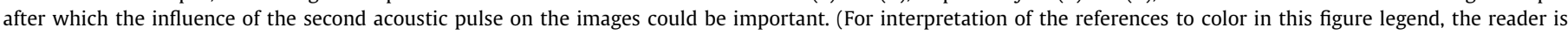
referred to the web version of this article.)

\subsubsection{Argon compressed to $11.4 \mathrm{GPa}$ and $15.4 \mathrm{GPa}$}

For the argon sample, the in-depth dependency of the Brillouin oscillation frequency with respect to lateral positions is shown in Fig. 4 at pressure values of $11.4 \mathrm{GPa}$ (a) and $15.4 \mathrm{GPa}$ (b). The lateral distance between adjacent depth profiles is $4 \mu \mathrm{m}$. Here again the frequencies are represented by colors and their values are given in the corresponding color bars. The signals at both pressures are treated using the previously described STFT processing, though with a Hann window having temporal size of 60 ps at FWHM (nearly equal to the length of the longest Brillouin cycle). This window duration corresponds to an in-depth resolution of about $0.32 \mu \mathrm{m}$ at $11.4 \mathrm{GPa}$ [Fig. 4 (a)] and $0.36 \mu \mathrm{m}$ at $15.4 \mathrm{GPa}$ 
[Fig. 4(b)]. Considering the best in-depth resolution controlled by the chosen signal processing and the lateral dimensions of the pump-probe overlap at FWHM of $5.7 \mu^{2}$, the minimal optoacoustically tested volume was about $1.8 \mu \mathrm{m}^{3}$.

In the experiments with argon, contrary to the case of water ice, the time moment when the generated acoustic pulse reaches the argon/diamond interface is not visible in the frequency versus depth plots. Both from the decay of particular Brillouin oscillations in Fig. 2 and from the amplitude images presented in Fig. 4 (c) and (d), it follows that the signal at the Brillouin frequency completely vanishes after propagating in solid argon during $1 \mathrm{~ns}$, corresponding to the penetration depths of less than $6 \mu \mathrm{m}$. For comparison, the optically measured thickness of the sample at $11.4 \mathrm{GPa}$ is about $28 \mu \mathrm{m}$. This vanishing of the acoustic signal is due to a strong attenuation of the coherent acoustic pulses in argon, which was earlier noticed in the experiments in Ref. [25] where argon was used as a pressure-transmitting medium. On the other hand, as reported in Ref. [20] and discussed above, the signal cannot be processed accurately at the time close to the launching of the sound pulse by the opto-acoustical generator because of imperfect filtering of the thermo-reflectance contribution at $\mathrm{GHz}$ frequencies. Thus, to determine the depth coordinate, the choice has been made to consider that the acoustic pulse propagates at the mean velocity from time $t=0$ of its generation up to the time where the determination of the Brillouin frequency becomes sufficiently exact. This explains the starting non-zero depth value in the 2D images shown in Fig. 4(a) and (b).

An important observation that emerged from the analysis of the experimental data obtained for solid argon is that, under particular circumstances, the choice of the thickness of the optoacoustic generator/transducer could play an important role in the depthprofiling experiments. The pump laser pulse initially generates two acoustic pulses one of which starts to penetrate in the solid argon while another propagates inside the generator toward its interface with the diamond culet. The acoustic pulse propagating across a thin generator can thus be reflected at the generator/diamond interface and propagate back to the generator/argon interface where a second acoustic pulse is then launched in the solid argon. The second coherent acoustic pulse penetrating in the argon produces additional scattering of the probe light and, in general, causes degradation of the transient reflectivity signal and loss of in-depth spatial resolution of this imaging technique. This is because in the signal of transient optical reflectivity the information on the material properties from two spatially separated points will be mixed. However, under particular circumstances the second coherent acoustic pulse launched in the sample could be useful. For example, this could be the case, if the first acoustic pulse launched in the sample and the transient temperature related background in the optical reflectivity signal are both importantly attenuated at the delayed time moment of the second acoustic pulse launching. Then the picosecond acoustic interferometry signal from the second coherent acoustic pulse launched in the sample will provide a background-free Brillouin oscillation in the immediate vicinity of the optoacoustic transducer and thus an opportunity for a precise depth-profiling of material properties in this region, which in the case of the first acoustic pulse is strongly suffering from the temporal overlap of the Brillouin oscillation with transient heating-induced reflectivity variations. Potentially, the thickness and the material of the optoacoustic transducer could be selected/designed for the sample imaging with the second echo pulse. Further discussion of possible consequences of the acoustic pulses reverberation inside the optoacoustic transducer (ringing of the optoacoustic transducer) is beyond the scope of the present work. We just note that the above-presented general principles are preliminary confirmed by the experiments in argon, the results of which are presented in Figs. 2 and 4.
In fact we observed a significant and abrupt increase of Brillouin frequency in experiments conducted at $15.4 \mathrm{GPa}$ at depths around 2.3-2.5 $\mu \mathrm{m}$ [Fig. 4(b)]. This is not the signature of the in-depth layering of the sample, as it could have been tentative to suggest in the spirit of our observations in water ice [see Fig. 3(a) for example], but the manifestation of launching at the generator-argon interface of the second coherent acoustic pulse. This hypothesis is confirmed by the corresponding Brillouin amplitude image in Fig. 4(d) demonstrating that the amplitude of the coherent acoustic phonon is strongly attenuated before the time moment of around $0.4-0.5 \mathrm{~ns}$, which corresponds to the distances of abrupt increase of frequency in the Brillouin frequency images in Fig. 4(b). Using the values of sound velocities in iron from Ref. [32] we estimated that the time delay of $0.4-0.5 \mathrm{~ns}$ corresponds to approximately 2.7-3.4 $\mu \mathrm{m}$ propagation distance of coherent acoustic pulses. Thus $0.4-0.5$ ns time delay could correspond to the delay of the second acoustic pulse launched in argon relative to the first one in the generator of $1.3-1.7 \mu \mathrm{m}$ thickness. This estimate of the generator thickness is in accordance both with its measurements at ambient conditions before loading the generator in the DAC and also with the results of optical measurements of the thickness of the sample at high pressure in two neighbor lateral positions, one on the generator and another outside the generator. The data accumulated at 11.4 GPa are in favor of our hypothesis on various possible manifestations of the second acoustic pulse in argon imaging as well. Although in Fig. 4(a) the zone of the Brillouin frequency increase is less pronounced than in Fig. 4(b), it is still distinguishable at depths around $2.0-2.2 \mu \mathrm{m}$. At the same time the Brillouin amplitude images in Fig. 4(c) (see also the Brillouin oscillation in lower part of Fig. 2) are providing even more convincing picture of the acoustic echo arrival in argon around $0.5 \mathrm{~ns}$. We note that the above estimates are very roughly obtained through visible inspection of the images with an attempt to get an idea of the phenomena manifested in average over the lateral scan. For finding the precise correspondence between the echo-type features in the Brillouin amplitude images and the abrupt increase of the frequency in the Brillouin frequency images, the estimates should be done in each lateral point separately.

We conclude that our estimates and experiments indicate that the features in frequency images of argon at depth exceeding about $2.0-2.3 \mu \mathrm{m}$ are related to the launching of the second coherent acoustic pulse in argon. Note that in the experiments with water ice the thickness of the optoacoustic transducer was about 1.5 times larger than in the experiments with argon and we have not found in the images any features at the estimated delay time for launching the second acoustic pulse in $\mathrm{H}_{2} \mathrm{O}$, that could indicate the influence of the second pulse on the images. This could be because of either a stronger attenuation of the sound pulse in $\mathrm{Fe}$ at larger distances in comparison with those in the argon experiments or due to a significantly weaker attenuation of coherent sound in water ice at pressures around between 57 and $84 \mathrm{GPa}$ than in argon at pressures between 11.4 and $15.4 \mathrm{GPa}$, or to both.

In the current study we have not used "effect" of the second launched pulse to improve precision in evaluation of the material parameters, because the goal was to present qualitatively the richness of the information obtained using the presented laser ultrasonic imaging even without profiting from this effect. In Fig. 4 (a) and (b) the black lines stand for delimiting the depth after which the influence of the second acoustic pulse on the images could be important. To the right of the black lines not just the determined Brillouin frequencies but also the estimated depths are supposed to be biased/poisoned by the presence of the second coherent acoustic pulse in argon. The zone of our analysis in Fig. 4 (a) and (b) is to the left of the black lines, where the measured values are not affected by the second launched pulse. The frequencies at both pressures show strong changes in lateral direction some- 
Table 1

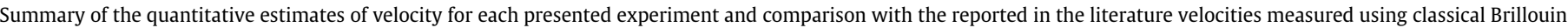
scattering.

\begin{tabular}{|c|c|c|c|c|c|}
\hline Pressure (GPa) & Average velocity $(\mathrm{km} / \mathrm{s})$ & Window size at FWHM (ps) & Density $\left(\mathrm{kg} / \mathrm{m}^{3}\right)$ & Refractive index at $800 \mathrm{~nm}$ & Velocity from the literature $(\mathrm{km} / \mathrm{s})$ \\
\hline \multicolumn{6}{|l|}{ Argon } \\
\hline 11.4 & $4.8 \pm 0.3^{*}$ & 66.5 & $3000^{\mathrm{a}}$ & $1.6 \pm 0.1^{b}$ & $\begin{array}{l}4.6-5.5^{\mathrm{a}} \\
4.2-5.4^{\mathrm{b}}\end{array}$ \\
\hline 15.4 & $5.5 \pm 0.3^{*}$ & 66.5 & $3300^{\mathrm{a}}$ & $1.6 \pm 0.1^{b}$ & $\begin{array}{l}5.1-6.0^{\mathrm{a}} \\
4.5-5.9^{\mathrm{b}}\end{array}$ \\
\hline \multicolumn{6}{|l|}{ Water ice } \\
\hline 57 & $12.5 \pm 0.1^{*}$ & 45 & $2770^{c}$ & $1.79 \pm 0.02^{\mathrm{c}}$ & $13 \pm 0.3^{\mathrm{d}}$ \\
\hline 84 & $14.5 \pm 0.1^{*}$ & 45 & $3120^{c}$ & $1.81 \pm 0.02^{c}$ & $15.5 \pm 0.3^{d}$ \\
\hline
\end{tabular}

\footnotetext{
* Indicated uncertainty of the average velocity is calculated from the uncertainty of the refractive index data only.

a Ref. [34].

b Ref. [33]

c Ref. [35].

d Ref. [36].
}

times even between two adjacent in-depth profiles, and smooth and similar at each separately taken lateral position when the indepth coordinate changes. Generally speaking, in the images of solid argon reported here in Fig. 4(a) and (b), the lateral texturing dominates over the in-depth one, while in the images of water ice in Fig. 3 the situation is opposite. At $15.4 \mathrm{GPa}$, the image shows clearly two regions, before and after the center of the generator in the lateral direction, with different mean frequencies. On the other hand, at $11.4 \mathrm{GPa}$, the strong frequency changes do not have similar distribution that could be clearly split into two parts. In order to check whether these differences in frequencies could come from non-hydrostatic loading of the DAC, we have estimated the changes in pressure that correspond to the equivalently strong changes in frequencies using the dependencies of the velocity and refractive index on pressure reported in Ref. [33]. Note that the refractive index of solid argon is weakly dependent on pressure in the considered pressure interval [33]. The estimates demonstrate that the pressure gradients needed to cause the corresponding frequency (or velocity) changes ( $1.56 \mathrm{GPa}$ per $4 \mu \mathrm{m}$ ) are an order of magnitude higher than what we have measured over the entire cell by means of the ruby fluorescence scale $(1.5 \mathrm{GPa}$ difference between ruby particles separated by about $45 \mu \mathrm{m}$ ). On the contrary, the longitudinal velocity anisotropy (estimated as the difference between the minimum and maximum velocity values) has been reported in Ref. [34] to be around $16 \%$ for pressures close to 11.4 GPa and 15.4 GPa. Considering the velocity anisotropy in our measurements, the highest relative change in the mean velocity between adjacent lateral positions is only $8 \%$ at $11.4 \mathrm{GPa}$ and $4 \%$ at $15.4 \mathrm{GPa}$. If we consider the velocity variations for a single lateral position, the largest difference is measured to be $12 \%$ at 11.4 GPa and $10 \%$ at $15.4 \mathrm{GPa}$. However, when considering the entire scanned volume, the global difference between the highest and the lowest observed frequencies was found to be $18 \%$ and $14 \%$ at pressures $11.4 \mathrm{GPa}$ and $15.4 \mathrm{GPa}$, respectively. These values are in the range of the expected $16 \%$ longitudinal velocity anisotropy [34]. So the differences in the local frequencies revealed by our laser ultrasonic imaging of solid argon can be attributed to the difference in the orientation of the crystallite groups inside the polycrystalline sample.

\subsection{Quantitative estimates of the acoustic velocities in compressed argon and compressed water}

The technique of time-domain Brillouin scattering that was applied by us here for imaging can be used similar to classic Brillouin scattering for the quantitative evaluation of the acoustic properties of materials at high pressures when this is required. As an example, a summary of the quantitative estimates of velocity for each presented case is now proposed. The average values are included in Table 1 and compared with velocities measured using classical Brillouin scattering and reported in the literature. The window sizes at FWHM used to process the experimental data for quantitative estimates were: $66.5 \mathrm{ps}$ for solid argon and $45 \mathrm{ps}$ for water ice. The average velocities for each of the samples at a particular pressure were obtained as follows. Primarily, the complete set of signals, for instance, 14 signals for argon $11.4 \mathrm{GPa}$ experiment, measured in a sample were processed using STFT approach with the mentioned window size. Secondly, the frequency values coming from the noisy signal parts together with parts corresponding to a second acoustic pulse, if any, were removed. These steps gave some tens of depth dependent Brillouin frequency values for each signal, which produce a few hundreds of Brillouin frequency values for the tested sample volume. Finally, these Brillouin frequency values from the whole tested volume were averaged and the velocity was calculated using Eq. (1) for a given sample at a particular pressure. The refractive index data were taken from the literature. It was considered that the angle between the propagation directions of probe and the acoustic pulse is equal to zero [20]. The wavelength of the probe beam is $804 \mathrm{~nm}$. The uncertainties in the indicated velocities stated in Table 1 are only due to the uncertainties in the reported values of the refractive index. The values of the average velocity obtained in this paper (Table 1 ) are in a very good agreement with values reported in the literature both for argon sample and water ice sample, at each pressure. Careful processing of the measured minimum and maximum frequencies could provide the values for the complete set of single crystal elastic constants, though this is beyond the scope of the present paper.

\section{Conclusion}

We have presented an experimental method of 2D imaging of polycrystalline water ice and solid argon aggregates compressed to high pressures in a DAC obtained by applying the technique of picosecond laser ultrasonic interferometry (time-domain Brillouin scattering). Our experimental results demonstrate that this technique is an effective tool for imaging the texturing/structuring of polycrystalline transparent aggregates providing a significantly better in-depth resolution when compared with the classical frequency-domain Brillouin scattering. Our experiments reveal differences in texturing of water ice and solid argon that definitely require more elaborated additional investigations in larger intervals of pressures.

\section{Acknowledgments}

This research was conducted in the frame of the "LUDACism" project supported by the ANR BLANC 2011 program. 


\section{References}

[1] C. Thomsen, J. Strait, Z. Vardeny, H.J. Maris, J. Tauc, J. Hauser, Coherent phonon generation and detection by picosecond light pulses, Phys. Rev. Lett. 53 (1984) 989, http://dx.doi.org/10.1103/PhysRevLett.53.989.

[2] I-J. Chen, P.-A. Mante, C.-K. Chang, S.-C. Yang, H.-Y. Chen, Y.-R. Huang, L.-C. Chen, K.-H. Chen, V. Gusev, C.-K. Sun, Graphene-to-substrate energy transfer through out-of-plane longitudinal acoustic phonons, Nano Lett. 14 (2014) 1317-1323, http://dx.doi.org/10.1021/nl404297r.

[3] Y-C. Chieh, Y-R. Huang, H-P. Chen, V. Gusev, C-K. Sun, Sub-THz coherent phonon coupling between solid and liquid water, J. Phys: Conf. Ser. 92 (2007). http://iopscience.iop.org/1742-6596/92/1/012025 012025.

[4] P.-A. Mante, C.-C. Chen, Y.-C. Wen, H.-Y. Chen, S.-C. Yang, Y.-R. Huang, I.-J. Chen, Y.-W. Chen, V. Gusev, M.-J. Chen, J.-L. Kuo, J.-K. Sheu, C.-K. Sun, Probing hydrophilic interface of solid/liquid-water by nanoultrasonics, Sci. Rep. 4 (2014), http://dx.doi.org/10.1038/srep06249 6249.

[5] T. Dehoux, M. Abi Ghanem, O.F. Zouani, J.-M. Rampnoux, Y. Guillet, S. Dilhaire, M.-C. Durrieu, B. Audoin, All-optical broadband ultrasonography of single cells, Sci. Rep. 5 (2015), http://dx.doi.org/10.1038/srep08650 8650.

[6] M. Xu, L.V. Wang, Photoacoustic imaging in biomedicine, Rev. Sci. Instrum. 77 (2006) 041101, http://dx.doi.org/10.1063/1.2195024.

[7] L. Wang, Photoacoustic imaging and spectroscopy, in: Optical Science and Engineering, CRC Press, Taylor \& Francis, 2009, ISBN 9781420059922, p. 518.

[8] P.C. Beard, Biomedical photoacoustic imaging, Interf. Focus 1 (2011) 602-631, http://dx.doi.org/10.1098/rsfs.2011.0028.

[9] T. Berer, A. Hochreiner, S. Zamiri, P. Burgholzer, Remote photoacoustic imaging on solid material using a two-wave mixing interferometer, Opt. Lett. 35 (2010) 4151-4153, http://dx.doi.org/10.1364/OL.35.004151.

[10] J. Gateau, T. Chaigne, O. Katz, S. Gigan, E. Bossy, Improving visibility in photoacoustic imaging using dynamic speckle illumination, Opt. Lett. 38 (2013) 5188-5191, http://dx.doi.org/10.1364/OL.38.005188.

[11] G. Langer, K.-D. Bouchal, H. Grün, P. Burgholzer, T. Berer, Two-photon absorption-induced photoacoustic imaging of rhodamine $\mathrm{B}$ dyed polyethylene spheres using a femtosecond laser, Opt. Express 21 (2013) 22410-22422, http://dx.doi.org/10.1364/OE.21.022410.

[12] R.W. Schoonover, M.A. Anastasio, Compensation of shear waves in photoacoustic tomography with layered acoustic media, J. Opt. Soc. Am. A: 28 (2011) 2091-2099, http://dx.doi.org/10.1364/JOSAA.28.002091.

[13] J. Tittelfitz, Thermoacoustic tomography in elastic media, Inverse Probl. 28 (2012), http://dx.doi.org/10.1088/0266-5611/28/5/055004 055004

[14] C. Thomsen, H.T. Graham, H.J. Maris, J. Tauc, Surface generation and detection of phonons by picosecond light pulses, Phys. Rev. B 34 (1986) 4129, http://dx. doi.org/10.1103/PhysRevB.34.4129.

[15] H.T. Graham, H.J. Maris, J. Tauc, Picosecond ultrasonics, IEEE J. Quantum Electron. 25 (1989) 2562, http://dx.doi.org/10.1109/3.40643.

[16] V. Gusev, A.M. Lomonosov, P. Ruello, A. Ayouch, G. Vaudel, Depth-profiling of elastic and optical inhomogeneities in transparent materials by picosecond ultrasonic interferometry: theory, J. Appl. Phys. 110 (2011) 124908, http://dx doi.org/10.1063/1.3665646.

[17] C. Mechri, P. Ruello, J.M. Breteau, M.R. Baklanov, P. Verdonck, V. Gusev, Depthprofiling of elastic inhomogeneities in transparent nanoporous low- $k$ materials by picosecond ultrasonic interferometry, Appl. Phys. Lett. 95 (2009), http://dx.doi.org/10.1063/1.3220063 091907.

[18] A. Steigerwald, Y. Xu, J. Oi, J. Gregory, X. Liu, J.K. Furdyna, K. Varga, A.B. Hmelo G. Lüpke, L.C. Feldman, N. Tolk, Semiconductor point defect concentration profiles measured using coherent acoustic phonon waves, Appl. Phys. Lett. 94 (2009), http://dx.doi.org/10.1063/1.3099341 111910
[19] A.M. Lomonosov, A. Ayouch, P. Ruello, G. Vaudel, M.R. Baklanov, P. Verdonck, L. Zhao, V.E. Gusev, Nanoscale noncontact subsurface investigations of mechanical and optical properties of nanoporous low- $k$ material thin film, ACS Nano 6 (2012) 1410-1415, http://dx.doi.org/10.1021/nn204210u.

[20] S.M. Nikitin, N. Chigarev, V. Tournat, A. Bulou, D. Gasteau, B. Castagnede, A. Zerr, V.E. Gusev, Revealing sub-mm and mm-scale textures in $\mathrm{H}_{2} \mathrm{O}$ ice at megabar pressures by time-domain Brillouin scattering, Sci. Rep. 5 (2015), http://dx.doi.org/10.1038/srep09352 9352.

[21] S. Danworaphong, M. Tomoda, Y. Matsumoto, O. Matsuda, T. Ohashi, H. Watanabe, M. Nagayama, K. Gohara, P.H. Otsuka, O.B. Wright, Threedimensional imaging of biological cells with picosecond ultrasonics, Appl. Phys. Lett. 106 (2015), http://dx.doi.org/10.1063/1.4918275 163701.

[22] W.C. Moss, J.O. Hallquist, R. Reichlin, K.A. Goettel, S. Martin, Finite-element analysis of the diamond anvil cell - achieving 4.6 Mbar, Appl. Phys. Lett. 48 (1986) 1258, http://dx.doi.org/10.1063/1.96996.

[23] S. Merkel, R.J. Hemley, H.-K. Mao, Finite-element modeling of diamond deformation at multimegabar pressures, Appl. Phys. Lett. 74 (1999) 656, http://dx.doi.org/10.1063/1.123031.

[24] R. Boehler, New diamond cell for single-crystal x-ray diffraction, Rev. Sci. Instrum. 77 (2006), http://dx.doi.org/10.1063/1.2372734115103.

[25] F. Decremps, L. Belliard, B. Perrin, M. Gauthier, Sound velocity and absorption measurements under high pressure using picosecond ultrasonics in a diamond anvil cell: application to the stability study of AlPdMn, Phys. Rev. Lett. 100 (2008) 035502, http://dx.doi.org/10.1103/PhysRevLett.100.035502.

[26] R. Cote, A. Devos, Refractive index, sound velocity and thickness of thin transparent films from multiple angles picosecond ultrasonics, Rev. Sci. Instrum. 76 (2005), http://dx.doi.org/10.1063/1.1900645 053906.

[27] P. Loubeyre, R. LeToullec, E. Wolanin, M. Hanfand, D. Hausermann, Modulated phases and proton centering in ice observed by X-ray diffraction up to 170 GPa, Nature 397 (1999) 503-506, http://dx.doi.org/10.1038/17300.

[28] T.S. Duffy, Earth science: probing the core's light elements, Nature 479 (2011) 480-481, http://dx.doi.org/10.1038/479480a.

[29] H.-K. Mao, J. Badro, J. Shu, R.J. Hemley, A.K. Singh, Strength, anisotropy, and preferred orientation of solid argon at high pressures, J. Phys.: Condens. Matter 18 (2006) S963, http://dx.doi.org/10.1088/0953-8984/18/25/S04.

[30] M. Grimsditch, P. Loubeyre, A. Polian, Brillouin scattering and three-body forces in argon at high pressures, Phys. Rev. B 33 (1986) 7192, http://dx.doi. org/10.1103/PhysRevB.33.7192.

[31] B. Journaux, R. Caracas, P. Carrez, K. Gouriet, P. Cordier, I. Daniel, Elasticity and dislocations in ice X under pressure, Phys. Earth Planet. Inter. 236 (2014) 10 , http://dx.doi.org/10.1016/j.pepi.2014.08.002.

[32] N. Chigarev, P. Zinin, L.-C. Ming, G. Amulele, A. Bulou, V. Gusev, Laser generation and detection of longitudinal and shear acoustic waves in a diamond anvil cell, Appl. Phys. Lett. 93 (2008), http://dx.doi.org/10.1063/ 1.3013587181905

[33] B. Chen, A.E. Gleason, J.Y. Yan, K.J. Koshi, S. Clark, R. Jeanloz, Elasticity, strength, and refractive index of argon at high pressures, Phys. Rev. B 81 (2010), http:// dx.doi.org/10.1103/PhysRevB.81.144110 144110.

[34] H. Shimizu, H. Tashiro, T. Kume, S. Sasaki, High-pressure elastic properties of solid argon to 70 GPa, Phys. Rev. Lett. 86 (2001) 4568-4571, http://dx.doi.org/ 10.1103/PhysRevLett.86.4568.

[35] C.-S. Zha, R.J. Hemley, S.A. Gramsch, H.-K. Mao, W.A. Bassett, Optical study of $\mathrm{H}_{2} \mathrm{O}$ ice to $120 \mathrm{GPa}$ : dielectric function, molecular polarizability, and equation of state, J. Chem. Phys. 126 (2007) 074506, http://dx.doi.org/10.1063/ 1.2463773.

[36] M. Ahart, M. Somayazulu, S.A. Gramsch, R. Boehler, H.-K. Mao, R.J. Hemley, Brillouin scattering of $\mathrm{H}_{2} \mathrm{O}$ ice to megabar pressures, J. Chem. Phys. 134 (2011), http://dx.doi.org/10.1063/1.3557795 124517. 